\title{
Los "efectos estructurantes" del transporte: mito político, mistificación científica
}

Publicación original: Offner, J. M. (1993). Les «effets structurants» du transport: mythe politique, mystification scientifique. L'Espace géographique, $22(3), 233-242$.

\section{Estefanía Szupiany \\ (traducción y nota introductoria) \\ Instituto de Humanidades y Ciencias Sociales del Litoral, Consejo Nacional de Investigaciones Científicas y Técnicas, Argentina}

\begin{abstract}
Resumen
Desde la revolución industrial del siglo XIX, la retórica del "impacto" y del "efecto inducido" de los transportes sobre la urbanización y la planificación no ha dejado de acompañar al desarrollo de las infraestructuras de comunicación. Sin embargo, la demostración de la validez de la relación no ha sido efectuada. Investigar la influencia de la puesta en marcha de un equipamiento sobre la economía de un territorio plantea enormes problemas metodológicos. Los trabajos empíricos rigurosos no muestran, en el mejor de los casos, sino una amplificación y una aceleración de las tendencias preexistentes. Si el mito de los efectos estructurantes perdura, es por el uso político que se hace de él en los procesos de decisión y en los procedimientos de evaluación ex ante de los grandes proyectos.
\end{abstract}

The "structuring effects" of transportation: political myth and scientific mystification

\footnotetext{
Abstract

Beginning with the industrial revolution during the 19th century, the rhetoric of "impact" and the "induced effect" of transportation on urban planning and development has continuously accompanied the development of communication infrastructures. However, a demonstration of the validity of this relationship has yet to be produced. Evaluating the influence of the operational implementation of a particular kind of equipment on the economics of a territory poses enormous methodological problems. Even the most rigorous empirical studies can only be used to confirm the amplification and acceleration of pre-existing tendencies. The persistence of the myth of "structuring effects" can in fact be explained by the political utilization of the notion for decision-making and in evaluation procedures at the outset of important urban projects.
}

\section{Palabras clave}

Asistencia en la toma de decisión Desarrollo urbano Metodología Transporte

\section{Keywords}

Assistance in decision-making Development

Methodology

Transportation

\section{Palavras-chave}

Assistência na tomada de decisão Desenvolvimento

Metodologia

Transporte

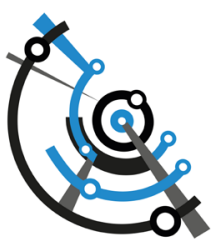




\section{Nota introductoria}

\section{"Mitos duros de matar"}

Veinticinco años atrás, la revista francesa L'Espace géographique publicaba un artículo que, de acuerdo con la opinión de algunos críticos y especialistas, hizo historia en los estudios del transporte. Bajo el título Les «effets structurants» du transport: mythe politique, mystification scientifique Jean-Marc Offner defendió su tesis enfocada en la relación entre el transporte y el territorio. El argumento central del escrito consistió en poner de manifiesto las deficiencias metodológicas de aquellos estudios que pretendían sostener la automaticidad -o efecto mecánico, repetitivo y previsible- del impacto de las infraestructuras de transporte sobre ciertos tipos de espacios, y en destacar el papel del mito de los efectos estructurantes en los discursos políticos y en los procesos de toma de decisiones. Si bien el modelo analítico basado en relaciones causales venía siendo objeto de revisión ya desde la década del sesenta (Miralles-Guasch, 2002), Offner reveló la continuidad del viejo paradigma, fundamentalmente en el ámbito político, en tanto mecanismo eficiente y operativo para la construcción de discursos de legitimación con base científica.

En el año 2014, en consonancia con el vigésimo aniversario de la publicación del artículo, L’Espace géographique decidió retomar la discusión en torno a los efectos estructurantes del transporte sobre el territorio (Offner et al., 2014). Entre los especialistas convocados, Offner introdujo la discusión manifestando tres explicaciones posibles acerca del éxito de su escrito en 1993: un título provocativo, una investigación multidisciplinaria, y un período de fuertes debates sobre proyectos de infraestructura. En este sentido, el autor sostiene que el intercambio entre el mundo académico y la sociedad civil se encargó de acelerar aún más la difusión del texto. Por su parte, Francis Beaucire (Offner et al., 2014:54-56) atribuye la trascendencia de aquella publicación en la medida en que Offner se dirigió tanto a la comunidad científica y al mundo de los expertos como a los representantes políticos.

Veinte años más tarde, afirma Offner, jel paradigma ha cambiado! La tendencia dominante en el mundo académico -entre geógrafos, economistas y especialistas del transporte- ya no es la creencia de que cualquier incremento en la infraestructura genera beneficios automáticos. Sin embargo, el viejo paradigma de la causalidad aún persiste en los círculos técnicos, políticos y periodísticos, alimentando discursos que legitiman determinadas acciones en el proceso de toma de decisión; en otras palabras, "Los mitos son duros de matar, porque continúan operando tanto como una explicación (demasiado) simple, como un punto de venta (engañoso) para los grandes proyectos" (Offner et al., 2014:53, traducción propia). Parafraseando al autor, dependerá, por lo tanto, de la comunidad científica asumir el rol de ampliar la gama de problemas y soluciones sometidos al debate público, permitiendo de este modo aumentar la maniobrabilidad de los actores políticos.

En función de esta convicción respecto de la importancia de la comunidad académica en su potencial vinculación con el mundo de las decisiones políticas, dos aspiraciones han incentivado la edición de la presente traducción. Por un lado, la de reivindicar un artículo clásico en el marco de los estudios del transporte y el territorio, hasta el momento sólo publicado en su idioma de origen. Por otro, la de fomentar la posible discusión de sus argumentos en el ámbito local, no para retomar el interrogante acerca de la existencia o no de los efectos estructurantes del transporte sobre el territorio, sino para reflexionar acerca de la pervivencia del mito en los discursos y las decisiones que subyacen a los procesos de construcción de nuestros espacios urbanos. 


\section{Dibliografía}

» Miralles-Guasch, C. (2002) Ciudad y transporte. El binomio imperfecto. Barcelona, Ariel.

»Offner, J. M., Beaucire, F., Delaplace, M., Frémont, A., Ninot, O., Bretagnolle, A. y Pumain, D. (2014). Les effets structurants des infrastructures de transport. L'Espace géographique 43 (1), 51-67. Disponible en <https://www.cairn.info/ revue-espace-geographique-2014-1-page-51.htm>.

\section{Estefanía Szupiany / eszupiany@gmail.com}

Arquitecta por la Universidad Nacional del Litoral y Doctoranda en Arquitectura por la Universidad Nacional de Rosario. Actualmente becaria doctoral CONICET y miembro del Instituto de Humanidades y Ciencias Sociales del Litoral IHUCSO UNL-CONICET. Docente en la Facultad de Arquitectura, Diseño y Urbanismo de la UNL e Investigadora en el Grupo de Trabajo CLACSO 2016-2019: Desarrollo, espacio y capitalismo global. 


\section{Los "efectos estructurantes" del transporte: mito político, mistificación científica}

\author{
Jean-Marc Offner
}

Ingeniero urbanista y analista político. Actualmente es Director General de a'urba, oficina de planificación urbana de Bordeaux métropole Aquitaine.

Efecto: aquello que es producido por una causa. Impacto: efecto de una acción fuerte, brutal. El veredicto del diccionario parece inapelable: es a una visión determinista de las relaciones entre las redes de transporte y las estructuras urbanas a las que hacen referencia las nociones de impacto y de efecto (inducido, estructurante).

De hecho, desde la revolución industrial del siglo XIX, este paradigma de la causalidad no ha dejado de acompañar al desarrollo de las infraestructuras de comunicación, del ferrocarril al tren de alta velocidad, de las carreteras a las autopistas, de los tranvías a los metros. Compartiendo el mismo credo saint-simoniano, políticos y científicos encuentran en el transporte, sino una garantía de progreso, al menos sí un factor explicativo de los modos de urbanización y planificación. ¿Pero la demostración de la validez de la relación ha sido efectuada? Nada es menos seguro. Y sin embargo, la relación de causa-efecto continúa suministrando un marco de análisis privilegiado en la economía de los transportes ${ }^{1}$, siendo aceptada con entusiasmo por el legislador local ${ }^{2}$ y por el periodista ${ }^{3}$.

Quisiéramos mostrar aquí que el uso poco circunspecto de la noción de "efecto estructurante" de los transportes constituye una suerte de mistificación científica a la vista de las lecciones de los trabajos empíricos y las reflexiones teóricas. Más a allá de un lamentable laxismo metodológico y de una imaginación conceptual deficiente, es sobre todo al uso estratégico de la retórica del efecto estructurante -verdadero mito político- al que debe atribuírsele el éxito de un pseudo-concepto, sin sustancia, pero parcialmente operativo.

\section{Mistificación científica}

El discurso científico de la socio-economía de los transportes sobre los "efectos" está rodeado de ambigüedad ${ }^{4}$. Incluso cuando la noción es generalmente rechazada, conserva un valor de uso y se incluye en los títulos de muchas publicaciones ${ }^{5}$. Es como si los méritos de las críticas enunciadas en su contra nos resultaran suficientes para privarla de toda credibilidad, habida cuenta de propuestas metodológicas y teóricas alternativas difíciles de asumir.

\section{Una noción sin fundamento}

Numerosos estudios empíricos ponen en duda la realidad de una causalidad lineal entre el desarrollo de una nueva oferta de transporte y las transformaciones espaciales, sociales o económicas. Con ello se refieren, más o menos explícitamente, a una visión sistémica ${ }^{6}$ del cambio.
1. Las referencias científicas y políticas se aplican en este texto esencialmente a Francia. Pero las observaciones que pueden realizarse a partir de los coloquios internacionales parecen indicar una gran convergencia de enfoques: ver la omnipresencia de los términos "impacto" y "efecto" en los títulos de las comunicaciones de las conferencias mundiales sobre la investigación de los transportes (Yokohama, 1989; Lyon, 1992). Ver también un informe reciente del Transport and Road Research Laboratory (Gran Bretaña) D. A. Walmsley y K. E. Ferett, The effects of rapid transit on public transport and urban development, 1991. 2. La LOTI (Ley de Orientación de los Transportes Interiores) de diciembre de 1982 formaliza las nociones de evaluación y revisión de los grandes proyectos de infraestructura y de las grandes decisiones tecnológicas. Ver el prospecto del Ministerio de Equipamiento: Évaluation économique et sociale des projects routiers inteurbains. Guide de recommandations, SETRA, mayo 1998.

3. Muy sorprendente es, por ejemplo, la interpretación efectuada por los periodistas de Le Monde sobre un estudio realizado acerca de los criterios de localización de las empresas. El artículo se titula: “Les entreprises en quête d'un nouveau site privilégient la qualité des route et celle des communications" (Le Monde, 5-6 de abril de 1992), en tanto que los resultados del estudio también destacan la importancia del potencial de mercado o la existencia de edificios adaptados... 4. Ejemplo sorprendente: a partir de la constante ausencia de nuevos polos de actividad en torno a las estaciones de TGV de CreusotMontchanin y de Mâcon-Loché, los participantes del primer coloquio de la Asociación de Ciudades Europeas TGV concluyen: "Se trata de dos estaciones nacidas de los primeros enlaces TGV, en 1981, en una época en la que casi nadie era consciente del impacto del TGV sobre el tejido económico y urbano" (TGV et aménagement du territoire, Syros Alternatives, 1991, p. 70). ¡No podríamos expresar mejor, a la vez, la creencia y las dudas acerca de la noción de impacto!

5. Es asombroso constatar, por ejemplo, que François Plassard, muy crítico de la noción de efecto, continúe utilizando el término, o palabras similares, en la mayor parte de los títulos de sus artículos, desde "La détection des effects structurants d'autoroute" (en colaboración con A. Bonnafous y D. Soum),

Revue économique, n. 2, 1974, hasta "L'impact territorial des transports à grande vitesse", en Pierre-Henri Derycke (ed.), Espace et dynamiques territoriales, Economica, 1992.

6. Obras de referencia en francés: Bernad Walliser, Systèmes et modèles, Le Seuil, 1997; JeanLouis Le Moigne, La Théorie du système général, PUF, 1977, 1984. 


\section{Dudas empíricas}

Con respecto a los transportes públicos urbanos, abundan los trabajos monográficos que intentan determinar los "efectos" de la puesta en servicio de una conexión ferroviaria. Efecto sobre el valor de las propiedades: en Villeurbanne, en la zona metropolitana de Lyon, la llegada del metro no modificó significativamente los precios inmobiliarios ${ }^{7}$; estos barrios de tradición obrera se habían transformado ya algunos años antes en zonas de vivienda para ejecutivos que trabajaban en el centro terciario de Lyon. Efecto sobre el volumen de negocios: encuestas realizadas luego de la apertura de estaciones de metro en los suburbios de Lyon y París $^{8}$ señalan únicamente un fenómeno de estimulación de las posiciones adquiridas. Se halla esta misma dinámica de amplificación y aceleración de tendencias preexistentes a través de la observación de la evolución de los centros de las ciudades francesas en relación con la creación de zonas peatonales ${ }^{9}$. Estos ordenamientos no hacen más que inscribirse en un contexto general de mutación urbana.

Una célebre investigación sobre el BART de San Francisco concluye que "los transportes públicos en general y el BART en particular no han tenido sino impactos menores sobre la decisión de localización de la mayor parte de las empresas", que "el BART no ha entrañado ninguna redistribución de las oficinas en la región", y que, "en resumen, el BART prácticamente no ha producido los beneficios económicos esperados por sus impulsores" 10 .

El análisis de las infraestructuras interurbanas suscita interpretaciones similares, ya se trate de la ferroviaria o la vial. Las conclusiones de François Plassard respecto de las autovías merecen citarse: "La visión simplista de mecanismos de causa-efecto no puede ser mantenida desde que se estudian las relaciones entre autovía y desarrollo regional [...] Es necesario afirmar con claridad que las declaraciones sobre los beneficios de las autovías, numerosas entre los políticos, no reposan sobre ningún fundamento científico [...] La introducción de la noción de potencialidad parece ser una de las vías eficaces que permiten este cambio de concepción" ${ }^{11}$. De investigaciones más recientes, llevadas a cabo con la participación del Laboratorio de Economía de los Transportes de la Universidad de Lyon II sobre el TGV, se desprenden conclusiones similares: "Se observa una brecha entre los cambios importantes y casi inmediatos introducidos por el TGV en la movilidad de las personas [...] y la lenta aparición de los efectos llamados "estructurantes" que se le podrían imputar. Las repercusiones del TGV sobre las actividades de las zonas comprendidas [por su servicio] no son automáticas [...]. Por lo tanto, conviene liberarse de todo discurso que establezca una relación determinista..." ${ }^{12}$.

Ante tales resultados, a veces se expresa la opinión de que la "muestra" de la observación geográfica es inadecuada ${ }^{13}$, o que el retroceso temporal es insuficiente para hacer aparecer los "efectos", indetectables a corto plazo. Sin embargo, los trabajos históricos invalidan esta tesis ${ }^{14}$. Montreuil, una comuna de los suburbios parisinos conectada por el metro desde 1937, ha sido comparada en la evolución de sus variables sociodemográficas y urbanísticas con comunas semejantes, pero alcanzadas por esa red algunas décadas más tarde. Ninguna diferencia ha podido ser puesta en evidencia ${ }^{15}$. En un período casi de doscientos años, han sido investigadas las correlaciones entre la existencia de una cobertura ferroviaria y el crecimiento económico de las pequeñas ciudades francesas de entre 5000 y 20000 habitantes ${ }^{16}$. Ninguna interdependencia ha sido detectada. La lista de ejemplos podría ampliarse sin dificultad para contradecir la existencia de efectos estructurantes, de consecuencias "mecánicas" (por lo tanto, repetitivas y previsibles) de la implantación de ciertos tipos de infraestructura sobre ciertos tipos de espacios.
7. Études de suivi des ouvertures des métros de Lyon et Marseille, CETUR, 1979.

8. J.-M. Offner, B. Marchand, L. Sanders, P. Chan, Transports collectifs et activités commerciales locales, rapport de recherche IRT, n. 57, abril 1982.

9. Les effets induits des zones piétonnes, CETUR, CETE d'Aix, STU, mayo 1980.

10. Citado por Christian Lefevre, "Transports collectifs et croissance urbaine: l'exemple des métros américains", Transports, n. 300, enero 1985. Ver también Robert L. Knight, "The impact of rail transit on land use: evidence and a change of perspective", Transportation, n. 9, 1980.

11. François Plassard, Les autoroutes et le développement régional, Presses universitaires de Lyon / Economica, 1977.

12. Effets socio-économiques du TGV en Bourgogne et RhôneAlpes, DATAR, INRETS, OEST, SNCF, LET, junio 1986.

13. "A propósito de los vínculos entre urbanización y transporte, parece que existe una contradicción entre el fenómeno macroscópico, donde el vínculo es indiscutible, y el fenómeno microscópico, donde el vínculo es menos evidente". Pierre Merlin, citado par Michel Gérard, "Transports et urbanisme", Temps libre, n. 10, otoño 1984.

14. Leer el magistral estudio de François Plassard, resumido en “Infrastructures de transport et transformation de l'espace. Le cas de la région du Creusot et de Montceau-les-Mines entre 1780 et $1980 "$, Culture technique, n. 19, marzo 1989.

15. Michel Rajchman, L'impact du prolongement du métro à Montreuil. Étude rétrospective, IAURIF, abril 1980.

16. Dominique Larroque, Gérard Jigaudon, Petites villes et infrastructures de transport, 1851-1954, Conservatoire national des arts et métiers, CDHT, 1985. 


\section{Críticas teóricas}

Esta condena inductiva de la noción de efecto reúne muchos de los logros de las teorías del cambio social. Son, en primer lugar, los filósofos y los sociólogos de la técnica quienes relativizan fuertemente, o incluso refutan, la idea del determinismo tecnológico ${ }^{17}$. Patrice Flichy lo afirma a propósito de las "máquinas de comunicar": "La historia de una invención es la de una serie de desplazamientos técnicos, sociales, pero en igualdad entre la técnica y lo social"18. Los dispositivos técnicos son construcciones sociales. La articulación histórica entre la extensión de una red de tranvías en Los Ángeles, desde finales del siglo XIX, y la dispersión simultánea de los suburbios ilustra bien esta cuestión. Frente a la tesis según la cual "los tranvías hicieron la ciudad"19, conviene subrayar que "esta nueva organización de la ciudad se inscribe en una representación del hábitat que aparece hacia mediados del siglo XIX en los Estados Unidos: la idealización de la casa individual en un ambiente bucólico. Este proyecto no fue inventado por las nuevas técnicas de transporte, pero fueron ellas las que lo hicieron posible"20.

En segundo lugar, los teóricos del cambio social abundan en este mismo sentido, ¡Marx incluido! ${ }^{21}$. Estos señalan la capacidad del interaccionismo para explicar las transformaciones de la sociedad ${ }^{22}$. Los prospectivistas hacen lo mismo, subrayando que "la mayor parte de los factores que las ciencias sociales toman en consideración están en interacción, es decir, que sus efectos varían según los valores de los otros factores a los cuales se encuentran vinculados, por lo tanto, según las situaciones consideradas" 23 . En un texto de referencia, Yves Barel subraya que "es ilusorio e ingenuo privilegiar un factor (la técnica, por ejemplo) para intentar construir una cadena causal en la que la técnica moldeara lo humano y lo social. No se regresa por ello a una teoría hueca y tautológica de la interacción universal, pues nada impide, al interior del sistema, estudiar las diferencias de los dinamismos específicos" 24 .

El mismo Fernand Braudel, a menudo invocado por los geógrafos o los economistas espaciales por sus análisis sobre la "larga duración" de la constitución de los territorios a partir de las redes, alerta a sus lectores contra una visión de la historia fundada sobre el determinismo de las infraestructuras, contra la creencia en la importancia de los "acontecimientos viales". "Sobre todo, no exageremos los acontecimientos de la historia vial. Ellos surgen, se contradicen, a menudo se desvanecen. Si los escucháramos, explicarían todo [...] En cuanto a la crónica vial de corta duración, la coyuntura por lo general ha distribuido con anticipación éxito o fracaso, flujo o reflujo" ${ }^{25}$. Fiel a esta tesis, Claude Harmelle ${ }^{26}$ formaliza la propuesta: "La ruta, los medios de transporte, no son ni el fundamento ni los motores del intercambio y de la buena fortuna, sino, por el contrario, sobre un espacio cualquiera, cuando preexisten motivos de intercambio, polos de producción, un deseo extrínseco [...], entonces, las rutas se abren, los técnicos innovan, medios de transporte más o menos adaptados ven la luz". Y el autor refiere a los relatos de viaje de Arthur Young, quien recorrió Francia poco antes de 1789: "Recorriendo el camino real de París a Toulouse, se maravilló del estado de las rutas francesas. Ellas son las más bellas de Europa, dice él, las mejor construidas, las mejor mantenidas, las más extensas. Pero las encontró desesperadamente vacías de tráfico, mientras que los caminos lodosos e incómodos de la Inglaterra de la misma época estaban atestados de carruajes".

El conjunto de pruebas parece suficiente para dar crédito a la idea de que la noción de efecto estructurante de los transportes carece de fundamento científico. Y sin embargo... estudios e investigaciones continúan empleándola sin escrúpulos.
17. "Es en cada caso, y en cada nivel, una forma de sociedad, una estructura organizacional o de poder la que desea, y es en función de esos deseos, pero sobre todo en función de proyectos movilizadores, que libera la imaginación, que toma forma y se despliega una tecnología”. Haroun Jamous, Pandore, n. 4, junio 1979.

18. Patrice Flichy, Une histoire de la communication moderne, La Découverte, 1991. 19. Christian Lefèvre, “Où les tramways font la ville. Los Angeles", Annales de la recherche urbaine, n. 21, enero 1984.

20. Patrice Flichy, op. cit.

21. Jean-Jacques Salomon, Le Destin technologique, Balland, 1992. 22. Ver, entre otros, Henri Mendras y Michel Forsé, Le Changement social, A. Colin, 1983.

23. Benjamin Matalon, "Les limites de la prévision scientifique", en André-Clément Découflé (dir.),

Traité élémentaire de prévision et de prospective, PUF, 1978.

24. Yves Barel, Prospective et analyse de système, La Documentation Française.

25. Fernand Braudel, Civilisation matérielle et capitalisme, A. Colin, 1967. 26. Claude Harmelle, “Les piqués de l'aigle. Saint-Antonin et sa région (1850-1940). Révolutions des transports et changement social", Recherches, n. 47-48, 1982. Ver también la comunicación en el Seminario de Investigación SERTINRETS del 14 de junio de 1985 sobre Les Effets économiques et sociaux des aménagements de transports. 


\section{Advertencias poco atendidas}

Abandonar un paradigma no es algo simple. Como lo recuerda Thomas Kuhn, "ocurre en las ciencias como en la industria: la renovación de las herramientas es un lujo que debe reservarse para las circunstancias que lo exijan. La crisis significa que nos encontramos frente a la obligación de renovar las herramientas"27. Sin dudas, nos encontramos en una fase de transición. La crisis comienza a ser percibida allí donde el modelo de la causalidad lineal produce, cada vez más visiblemente, incoherencias importantes en la coordinación entre las políticas de transporte y de planificación ${ }^{28}$. Pero, utilizadas sin gran cuidado epistemológico, los instrumentos tradicionales de análisis de la interacción flujo-espacio continúan engañando. En fin, las soluciones metodológicas y conceptuales de reemplazo continúan en un estado de elaboración insuficiente.

\section{Un rigor metodológico deficiente}

Es inútil detenerse en el silogismo clásico que confunde concomitancia y correlación, y luego, correlación y relación de causa-efecto. Sólo hay una evaluación comparativa ${ }^{29}$, cuya versión más acabada se encuentra en el ámbito de la farmacología ${ }^{30}$. Aun así, es necesario saber comparar la situación observada. Numerosas equivocaciones de interpretación provienen, por ejemplo, de una comparación apresurada entre un "antes" (antes de la infraestructura de transporte) y un "después", como si "todas las cosas en igualdad de condiciones" pudieran tener un único sentido ${ }^{31}$. Esto ha conducido a aporías sorprendentes, como la de la constatación de un aumento del consumo energético a partir del desarrollo del transporte público. Se ha mostrado que es conveniente recurrir al método de los escenarios ${ }^{32}$ para comparar la situación del "después" con un estado virtual del "después" sin la infraestructura de transporte.

Otro error frecuente consiste en querer "aislar" la variable "transporte", en tanto que una visión sistemática del cambio invita, por el contrario, a interesarse en los procesos de agregación, de sinergia, de interdependencia, etc. ${ }^{33}$. Así es como se descontextualiza la infraestructura de transporte de las condiciones políticas, económicas y sociales que han permitido su realización, y de los fenómenos de apropiación que ella entraña. La reificación del objeto técnico (el TGV, el metro, la autopista...) olvida que el equipamiento considerado no es una "cosa", una realidad estática, sino un soporte de acciones. Por lo tanto, se habla de evaluación de proyectos, mientras que sólo la evaluación de políticas parece pertinente.

Estas incomprensiones encuentran al menos una parte de su explicación en el deslizamiento semántico operado implícitamente entre el entorno físico y el entorno socio-económico. En el primer caso, es conveniente investigar, a través de "estudios de impacto", los efectos de un equipamiento sobre el medio natural. Ocurre todo lo contrario con el segundo. Un sistema espacial, con su espesor político, económico, social, es susceptible de anticipación, de desviaciones, de táctica y de estrategia. La transferencia hacia las ciencias sociales del modelo de causalidad lineal, aceptable en toda primera aproximación de las ciencias llamadas exactas, conduce a un callejón sin salida.

\section{Una inventiva conceptual para estimular}

Este diagnóstico nos alentó, hace algunos años, a proponer la comprensión de la nueva oferta de transporte, no sólo como un factor de amplificación y aceleración de tendencias preexistentes, sino también como un instrumento potencial de las estrategias de los actores territoriales, como una oportunidad de acción relativizada por el grado de "mutabilidad" de las variables relacionadas ${ }^{34}$. Por más importante que fuera, una infraestructura de transporte no podría modificar el uso del espacio si los dispositivos reglamentarios del derecho urbano impidieran, por ejemplo, toda
27. Thomas S. Kuhn, La Structure des révolutions scientifiques, Flammarion, 1983. 28. El tópico de la necesaria coherencia entre transporte y urbanismo, del carácter integral de las demandas, es un leitmotiv en el ámbito profesional de los transportes. Ver, entre otros ejemplos, el del Coloquio de la Federación Nacional de Agencias de Urbanismo: Urbanisme, déplacements, transports, Lyon, octubre 1981.

29. Con algún retraso respecto de la edición alemana o americana, la literatura francófona sobre las cuestiones de evaluación conoce hoy una rápida expansión. Ver, última obra a la fecha, el Rapport annuel sur l'évolution des pratiques d'évaluation des politiques publiques del Consejo Científico de Evaluación: L'évaluation, de l'expertise à la responsabilité, La Documentation Française, diciembre 1991.

30. Daniel Schwartz, "Les techniques d'évaluation en épidémiologie", en Jean-Pierre Nioche, Robert Poinsard (dir.), L'Évaluation des politiques publiques, Economica, 1984. 31. Concluyendo su estudio de "seguimiento" del metro de Lyon, Alan Bonnafous, Danièle Patier-Marque y François Plassard reconocen que "la mayor parte de las modificaciones observadas en las dos investigaciones son raramente del todo ajenas al metro, aunque tampoco se encuentran totalmente ligadas a él. Ocurre simplemente que todas las cosas no se encuentran en igualdad de condiciones entre el antes y el después del metro". Mobilité et vie quotidienne, Presses Universitaires de Lyon, 1981. 32. Jean-Pierre Orfeuil, "La promotion des transports collectif urbains dans le contexte énergétique actuel", Transports, n. 252, abril 1980.

33. Ver los aportes de sociólogos americanos como Robert Axelrod, Manur Olson, Thomas Schelling y, naturalmente, James March y Herbert Simon. En el plano empírico, Maurice Chevallier muestra en sus investigaciones sobre L'Impact du métro de Lyon sur les comportements et les modes de vies de ses habitants cómo las evoluciones en la movilidad de los individuos están subordinadas a la simultaneidad de una transformación de la oferta de transporte con una ruptura en el ciclo de vida y a la aparición de nuevos lugares a frecuentar. Ver Jean-Marc Offner, Brigitte Laurent, Maurice Chevallier, Aspects méthodologiques des études de suivi, rapport de recherche IRT, n. 77, junio 1985.

34. Roger Chaix, Michel Rajchman, Mutabilité du tissu urbain le long de la ligne ferrée de grande ceinture, IAURIF, 1980. 
modificación del coeficiente de ocupación de los suelos. La baja "cotización" de los terrenos que rodean a numerosas estaciones de metro o de la red del expreso regional en la región de París se explica por políticas municipales hostiles a la especulación inmobiliaria. Del mismo modo, las nuevas ofertas de transporte podrían no modificar sensiblemente los comportamientos de desplazamiento si los potenciales usuarios no las percibieran como una "ganga" para aprovechar, susceptible de integrarse en el proyecto individual o familiar en curso. Se produce, pues, la interacción entre dinámicas estructurales, en las que los proyectos de transporte se inscriben indefectiblemente, y las estrategias de actores posicionándose, a priori (si ellos son colaboradores o interlocutores de la decisión) o a posteriori, en relación con sus planes, otorgándoles un sentido que no se muestra ni inmutable ni exclusivo ${ }^{35}$.

En parte hallamos estas ideas en la reflexión según la cual el transporte representa una "condición necesaria pero no suficiente" (del crecimiento económico, del ordenamiento territorial, etc.). En la actualidad, regularmente se hace mención a la ausencia de efectos "automáticos" y a la necesidad de elaborar medidas de acompañamiento ${ }^{36}$. Pero conviene formalizar los conceptos que dan cuenta de esta modelización de las relaciones entre los transportes y la organización socio-económica del espacio. Tomar prestada la noción de congruencia ${ }^{37}$ del lenguaje corriente (y de modo más indirecto del de las matemáticas) forma parte de esta ambición.

En sentido estricto, la congruencia remite a la idea de homología estructural, utilizada implícitamente por Max Weber, entre otros. "Un fenómeno es explicado, no por la puesta en evidencia de un conjunto de causas y circunstancias históricas, sino por la puesta en evidencia de un paralelismo entre dos «estructuras» "38. Nos liberamos, de esta manera, del vínculo de causa-efecto (del huevo y la gallina...) en favor de un modelo de adaptación recíproca: el TGV no provoca la creación de parques tecnológicos, pero es el medio de transporte que mejor corresponde, en un momento dado, a la aparición de nuevas formas de organización espacial de las empresas innovadoras. Los transportes públicos urbanos no valorizan ni desvalorizan los centros de las ciudades, pero constituyen un elemento de tipo europeo de la centralidad urbana.

Se comprende fácilmente, no existe una congruencia sino históricamente datada; por lo tanto, relativa. La línea ferroviaria de Sceaux (actual sección sur de la línea B de la RER) poseía en su origen una funcionalidad interurbana; permitía a los parisinos adinerados ir esporádicamente a la campiña. Durante el período de entre-guerras, esta misma línea facilitó el desarrollo de urbanizaciones, ya que los obreros podían encontrar en los suburbios mejores condiciones de vivienda que en el París intra-muros gracias a la reducción del tiempo de trabajo, en parte reasignado en tiempo de transporte. Después de 1945, y con la electrificación de la línea, fueron las residencias colectivas para ejecutivos las que se construyeron en la proximidad de las estaciones del RER; proximidad no inmediata, puesto que un primer círculo de casas individuales ocupaba los alrededores más cercanos, pero el automóvil individual permitía entonces esta "devaluación"39. La misma línea ferroviaria se inscribe, pues, en dos "congruencias": camino ferroviario-reducción de tiempo de trabajo-casa individual-obreros; RER-valoración económica y social del suburbio sur-ejecutivos-vivienda colectiva ${ }^{40}$.

Sin duda, la noción de congruencia puede contribuir a una mejor comprensión del pasado y a una proyección más inteligente del futuro. Pero es verdad que da cuenta mejor de las "tendencias estructurales preexistentes" en las cuales se integran los proyectos de transporte, que de los aspectos de las estrategias de los actores. ¿No hay una pista para reflexionar en torno a la idea de la existencia de discrepancias temporales, de ritmos diferenciados, entre una congruencia macrogeográfica de largo plazo y las traducciones locales a corto plazo que proponen las instituciones y los actores económicos implicados? Nos detendremos aquí, en la sugerencia.
35. El metro de Lyon, de nuevo, yel reciente tranvía Bobigny-Saint-Denis, en el suburbio parisino, constituyen dos ilustraciones. Ver Alain Bieber, "Le rôle des transports en commun dans la planification de l'agglomération lyonnaise", en Gabriel Dupuy (dir.), Réseaux territoriaux, Paradigme, 1988 . Jean-Marc Offner, "Le tramway SaintDenis-Bobigny, entre réseaux et territoires", RTS, INRETS, n. 29, marzo 1991. 36. Ver el informe del Consejo General de Puentes y Caminos de noviembre de 1988, que presenta los Éléments de réflexion sur les mesures d'accompagnement des grandes infrastructures de transport, y el documento del SETRA sobre Les effets socioéconomiques des grandes infrastructures routières, junio 1988. También deben citarse las conclusiones del coloquio TGV et aménagement du territoire (op. cit.): "Jean Frébault, director de Arquitectura y Urbanismo del Ministerio de Equipamiento, llama la atención de los [miembros] electos sobre este posible espejismo:

«Es necesario prestar mucha atención al mito de los beneficios automáticos del TGV, o de una estación TGV en alguna ciudad, sobre su desarrollo económico. Sabemos que no hay un efecto sistemá tico sino hay una verdadera dinámica local, y es la responsabilidad de los [representantes] electos y de los actores económicos el ponerla en marcha" El TGV acelera o amplifica las situaciones favorables o desfavorables. No las crea a partir de la nada. El desarrollo del efecto TGV al nivel de una ciudad o de una región depende muy largamente de la oferta que se establezca y del dinamismo de los actores locales. En otras palabras, si no existe una estrategia real de desarrollo para explotar la ventaja del TGV, este último no solo no será de ninguna ayuda, sino que, por el contrario, corre el riesgo de convertirse en una desventaja. En efecto, como lo han señalado numerosos participantes, el TGV actúa a la vez como una bomba de empuje y como una bomba de succión. Puede traer riquezas, pero puede igualmente contribuir a vaciar a una región de su potencial de materia gris en provecho de una metrópoli más dinámica". 37. La noción de congruencia fue utilizada por primera vez por Jean-Marc Offner en noviembre de 1980: "L'évaluation des investissements de transport: effets ou congruence", coloquio del GRECO CNRS «Transport et Espace» sobre el tema Études de suivi et processus de décision. Pierre Merlin hizo referencia a ella en 1991, en su obra Géographie, économie et planification des transports (PUF). En 1992, Patrick Bonnel, en el LET, la reutilizó para un proyecto de estudio de seguimiento de la línea D del metro de Lyon.

38. Raymond Boudon, Les Méthodes en sociologie, Paris, PUF, coll. "Que sais-je?», 1973. 39. T. H. Moreau, La Ligne dans la vallée, Histoire et fonctionnement de la ligne de Sceaux, rapport Réseaux 2000, RATP, 1985

40. Ver Pierre Merlin, Les Transports urbains, Paris, PUF, coll. "Que sais-je?», 1992. 
En cualquier caso, los avances conceptuales permanecen todavía muy parciales para ofrecer un corpus de substitución sólido, incluso si es posible enunciar algunas recomendaciones metodológicas: la necesidad de un diagnóstico "en la dinámica" de las situaciones observadas, de una evaluación del grado de mutabilidad de los entornos, etc. ${ }^{41}$. Pero incluso más que esa ausencia, esperemos que temporal, de conceptos y de métodos fácilmente identificables y utilizables, es a su uso político al que la noción de efecto-transporte debe su excepcional longevidad.

\section{Mistificación política}

Si la retórica del efecto pertenece, en el plano científico, al mundo de la evaluación a posteriori, está mucho más vinculada, en el plano político, a los procedimientos de evaluación a priori de los proyectos de transporte. El político busca en el científico las razones de su acción, el científico intenta responder al pedido del político; los roles se ven reforzados por la legitimación recíproca...

\section{El "orden" técnico-político}

Tanto los representantes locales como los técnicos que "asisten en la decisión" adhieren generalmente con entusiasmo al paradigma de la causalidad lineal, la que los métodos de la evaluación técnico-económica de los proyectos suponen.

\section{Un discurso recurrente}

Las alusiones de la introducción ya lo han indicado: el "efecto" tiene buena prensa porque permite decir cosas simples, ofrecer explicaciones unívocas. Sería fastidioso establecer la nómina de sus ocurrencias. Es más interesante constatar que el efecto estructurante de los transportes deviene un leitmotiv desde finales del siglo XIX, cuando nacen y se desarrollan con rapidez la mayor parte de las redes técnicas modernas que conciernen a los transportes, a la energía o a las telecomunicaciones. Los treinta años de conflicto -entre la ciudad de París, por un lado, y las compañías ferroviarias y el Estado, por la otra ${ }^{42}$ - que preceden a la decisión y a la construcción del metro, se fundan sobre el a priori de que una red metropolitana a escala regional provocaría la huida residencial de los parisinos hacia la periferia. Los debates sobre el tranvía son del mismo orden ${ }^{43}$. A partir del examen de los censos de principios del siglo XX, la constatación de la simultaneidad del crecimiento de los suburbios cubiertos por el tranvía y la desaceleración del crecimiento parisino centraron la atención de las autoridades sobre la creación de líneas de transporte público. "Muy precozmente, incluso antes de ser explícitamente mensurable, la relación de causaefecto entre transporte público y éxodo parisino hacia los suburbios es del orden del lugar común"44. ¿Es necesario recordar aquí las batallas épicas libradas por los representantes locales a fin de que sus ciudades dispusieran de un activo decisivo: una estación de TGV, un cruce de autopistas? La actualidad procura cada día ejemplos suplementarios. Los metros, tanto en los Estados Unidos ${ }^{45}$ como en Francia, gozan del mismo prestigio.

\section{Una retórica determinista de la evaluación ex ante}

Las técnicas tradicionales de evaluación de proyectos, en la preparación de la decisión, participan en primer lugar de esta celebración del efecto. Los análisis de costobeneficio se atienen a tener cuenta, si es posible cuantificando y monetizando, los efectos directos e indirectos. Si bien no es cuestión de rehacer aquí la historia del cálculo económico ${ }^{46}$, es necesario constatar que las tentativas de modernización de
41. Jean-Marc Offner, “Aspects méthodologiques d'un suivi exploratoire du VAL sur deux quartiers lillois", Les Suivis des grandes infrastructures de déplacements urbains, CETUR, 1985.

42. Alain Cottereau, “L'apparition de l'urbanisme comme action collective. L'agglomération parisienne au début du siècle. I. De Haussmann à la construction du métropolitain", Sociologie du travail, n. 4, octubre-diciembre 1969.

43. Ver Dominique Larroque, "Transports urbains et transformations de l'espace parisien", Les Cahiers de l'IHTP, CNRS, n. 12, octubre 1989.

44. Anne Rasmussen, “Un discours à l'épreuve: politique des tramways et population de banlieue (1870-1914)", Villes en parallèle, n. 15-16, 1990.

45. Christian Lefevre, 1985, op. cit. 
estos enfoques (racionalización de las elecciones presupuestarias, análisis multricriterios, etc.) han conservado la lógica inicial del efecto, estimada por los ingenieroseconomistas ${ }^{47}$. Ya se hable de rentabilidad o de eficacia económica y social, el razonamiento de evaluación a priori privilegia la individualización de las consecuencias directas del proyecto considerado en detrimento de la consideración de las oportunidades que este representa como herramienta de una política. Surge de la deducción antes que de la retrodicción (en la que se supone realizado un fenómeno, el objetivo fijado, y se investigan las condiciones que lo volverían posible, los modos de alcanzarlo). Es por eso que continuamos evaluando sin reparos el interés de un proyecto de transporte en gran medida por los tiempos de desplazamiento que éste supone economizar, aunque también sabemos perfectamente que estas ganancias de tiempo encuentran, en un plazo más o menos breve, traducciones diversas: por ejemplo, ampliación de las fuentes de trabajo y extensión de las periferias, reestructuración de los programas de actividad.

La noción de evaluación, formalizada en Francia por la Ley de Orientación de los Trasportes Interiores de 1982, debería alentar la puesta en evidencia de tales discrepancias entre los criterios de evaluación a priori y la realidad de las evoluciones globales de las interacciones entre sistema de desplazamientos, sistema de localización y sistema de prácticas y relaciones sociales ${ }^{48}$. Sin embargo, postula la relevancia de las comparaciones antes-después, las que se supone que revelan cambios directamente atribuibles a la infraestructura de transporte.

\section{Un mito "operativo"}

Interesado en la noción de servicio público, un eminente politólogo afirmaba que ésta era un "mito legitimador", "una noción compleja en la cual se mezclan, de una manera indiscernible, indisociable e indisoluble, lo real y el mito, unidos por el cemento del derecho" ${ }^{\prime 9}$. Parafraseándolo, podría decirse que los efectos estructurantes de los transportes no son más que un mito operativo, en el que se asocian y se oponen la realidad de la interacción (¿de la congruencia?) y el mito de la causalidad, unidos por el cemento de los métodos de evaluación.

\section{Legitimación de la acción}

Cuando la Dirección de Transportes Terrestres del Ministerio de Equipamiento solicita a una oficina de estudio que proponga un método estándar de evaluación (ex post) de las operaciones de TCSP (transportes públicos en sitio propio) en las provincias, comprendemos que, más allá de la preocupación normal de una administración central por homogeneizar la recopilación de datos, el desafío es de orden argumentativo. Es necesario convencer (al ministro de Hacienda, al de gobierno) del interés para la comunidad en participar en el financiamiento de metros, de VAL, de tranvías... Los estudios de seguimiento deben interpretarse en términos similares, como herramientas de gestión exitosa ${ }^{50}$, de legitimación a posteriori de la acción. El discurso del efecto sirve para representar la racionalidad de la decisión política: "Tomo tal decisión porque conozco las consecuencias, y sé que ellas son positivas". Cómo evitar, habiendo llegado a este estado de reflexión, aplicar a la noción de efecto eso que Lucien Sfez dice de la decisión: “¿Por qué la decisión sobrevivirá a una «crítica de la decisión»? [...]. La función de la decisión es permitir que el actor actúe. [...] La decisión tiene por función fragmentar los actos estatales en otras tantas jurisdicciones respectivas" ${ }^{\prime \prime}$. El paralelismo es evidente: el mito del efecto autoriza y legitima la acción de quien toma las decisiones; permite la elaboración de proyectos sectoriales, a pesar de la evidencia de las interrelaciones entre políticas públicas.
47. Ver Jean-Marc Offnet, Marianne Ollivier-Trigalo (dir.), Les Grands projets de transports. Langages de l'évaluation. Discours de la décision, Synthèse INRETS, n. 10, noviembre 1987.

48. Esta formalización del sistema urbano en tres sub-sistemas fue propuesta por Alain Bonnafous et Hugues Puel en Physionomies de la ville, éd. Ouvrières, Économie et Humanisme, 1983.

49. Jacques Chevallier, Le Service public, PUF, coll. "Que sais-je?», 1987.

50. Ver el conjunto de contribuciones al coloquio del GRECO Transport et Espace. Études de suivi et processus de décision, CNRS, INRETS, noviembre 1980. Ver, como ilustración de esta función de los estudios de seguimiento, 1983-1988: six ans de métro dans la communauté urbaine de Lille, CETUR, 1990.

51. Lucien Sfez, Critique de la décision, Presses de la Fondation nationale des sciences politiques, 1987. 


\section{Incoherencias decisionales}

Sin embargo, no todo marcha bien en el mejor de los mundos, pues el mito operativo introduce algunas disfuncionalidades. "Diseccionando" las políticas de transporte por proyectos (efecto de extensión de línea, efecto de la reestructuración del sistema de autobús, efecto de la tarjeta de autobús, efecto del automatismo del metro), y siguiendo el famoso principio cartesiano vuelto obsoleto por la "complejidad" del mundo, minimiza las exigencias de coordinación: entre los diversos elementos del sistema de desplazamiento, entre las acciones de gestión de los flujos y de la ocupación del espacio, etc. Oculta la necesidad de medidas de acompañamiento ${ }^{52}$.

El mito político encuentra allí sus límites. No obstante, no es condenable. Su racionalidad instrumental justifica su existencia. La mistificación científica, por el contrario, no tiene nada para presentar en su defensa, excepto admitir la excusa de la pereza intelectual. La renovación de los paradigmas puede contribuir a una mejor gestión del cambio, que sepa tomar en cuenta el carácter dinámico y estratégico de las interacciones entre transporte y espacio. Pero el "científico" debe admitir que el discurso político posee su eficacia propia en las representaciones simplificadas de la realidad que desarrolla. Por lo tanto, debe considerar sin horror las virtudes del doble lenguaje.

\section{Agradecimientos}

Se agradece el permiso para la edición española del artículo Les «effets structurants» du transport: mythe politique, mystification scientifique a Jean-Marc Offner y a los editores responsables de la revista francesa L'Espace géographique; como así también al equipo editorial de la Revista Transporte y Territorio por su interés en la publicación de esta traducción. Por último, un especial agradecimiento a Manuel Tizziani por su revisión.
52. Frédéric Bellanger, Le TGV Atlantique au Mans, à SaintPierre-des-Corps, Tours et Vendôme: opportunités, acteurs, enjeux, Maison des Sciences de la Ville, Université de Tours, 1991. 